\title{
Consenso sobre el uso del monitor flash de glucosa de primera generación en pacientes menores de 18 años de edad con diagnóstico de diabetes mellitus tipo 1
}

\author{
Consensus on the use of the first generation flash glucose monitor in \\ patients under 18 years of age diagnosed with type 1 diabetes mellitus
}

Matallana $A M,{ }^{1}$ Céspedes $C,{ }^{2}$ Abad $V,{ }^{3}$ Durán $P,{ }^{4}$ Forero $C,{ }^{5}$ Martínez $L,{ }^{6}$ Lema $A,{ }^{7}$ Llano JP. ${ }^{8}$

\footnotetext{
${ }^{1}$ Pediatra endocrinóloga, Universidad del Valle. Profesora Asociada, Hospital Universitario del Valle. Cali, Colombia.

${ }^{2}$ Hospital Universitario San Ignacio, Endociencia, Universidad

Javeriana. Pediatra endocrinóloga, profesora asociada, Facultad de Medicina. Bogotá, Colombia.

${ }^{3}$ Pediatra endocrinóloga. Abad Laboratorio. Hospital Pablo Tobón Uribe. Medellín, Colombia.

${ }^{4}$ Pediatra endocrinóloga. Endociencia, Fundación Cardioinfantil-

IC. Profesora asociada, Universidad del Rosario y Universidad de la Sabana. Bogotá, Colombia.

${ }^{5}$ Pediatra endocrinóloga, Hospital Universitario Fundación Santa Fe, Hospital Universitario San Ignacio, Endociencia. Profesora ad honorem de la Pontificia Universidad Javeriana. Bogotá, Colombia. ${ }^{6}$ Pediatra endocrinóloga. CENPED N\&A SAS. Barranquilla, Colombia.

${ }^{7}$ Pediatra Endocrinóloga. Fundación Cardioinfantil-IC y Los Cobos Medical Center. Bogotá, Colombia.

${ }^{8}$ Pediatra endocrinólogo. Laboratorio de investigación hormonal. Bogotá, Colombia.
}

Autor de correspondencia: Audrey Mary Matallana Correo electrónico: lsardi@hotmail.com

Fecha de recepción: 2/03/2021

Fecha de aceptación: 15/03/21

\section{Resumen}

Introducción: la diabetes mellitus tipo 1 (DM1) es una de las patologías endocrinas más frecuentes en la población pediátrica. La glucometría capilar, método de autocontrol más recomendado, se asocia con molestias que pueden disminuir la adherencia, lo que impulsó la creación de nuevas tecnologías que respondan a las necesidades de los pacientes.

Objetivo: generar recomendaciones a partir del consenso de expertos acerca del uso del monitor flash de glucosa (MFG) en pacientes menores de 18 años con diagnóstico de DM1.
Materiales y métodos: se realizó un consenso tipo Delphi modificado en el que participaron 8 especialistas en endocrinología pediátrica y un grupo desarrollador. A partir del análisis de los resultados, se presentaron las recomendaciones.

Resultados: se obtuvieron recomendaciones acerca de indicaciones, frecuencia y modo de uso, perfil de seguridad e impacto de su uso en la calidad de vida.

Conclusiones: el MFG tiene una precisión comparable con la glucometría capilar y se considera un dispositivo seguro, con la capacidad de impactar positivamente en la calidad de vida de los pacientes y sus cuidadores.

Palabras clave: diabetes mellitus tipo 1, niño, adolescente, automonitorización de la glucosa sanguínea, sistema monitor flash glucosa.

\section{Abstract}

Introduction: Type 1 diabetes mellitus (DM1) is one of the most frequent endocrine pathologies in pediatric population. Capillary blood glucose testing, the most recommended selfmonitoring method, is associated with discomforts that could decrease adherence. This led to creation of new technologies that respond to patients' needs.

objective: To generate recommendations based on experts consensus about the use of the flash glucose monitor (FGM) in patients under 18 years of age with DM1 diagnosis.

Materials and methods: A modified Delphi-type consensus was carried out in which 8 specialists in pediatric endocrinology and a developer group participated. Based on the analysis of the results, the recommendations were presented.

Results: Recommendations about indications, frequency and mode of use, safety profile and impact of its use on quality of life were obtained. 
Conclusions: The FGM has a precision comparable with capillary glucose. It is considered a safe device, with the ability to impact positively the quality of life of the patients and their caregivers.

Keywords: Type 1 Diabetes Mellitus; Child, Adolescent; Blood Glucose Self-monitoring; Flash Glucose Monitoring System.

\section{Introducción}

La diabetes mellitus tipo 1 (DM1) es una de las patologías endocrinas y metabólicas más frecuentes en la población pediátrica. Se estima que 98200 niños menores de 15 años desarrollan DM1 a nivel mundial anualmente, y esta cifra aumenta a casi 128900 menores de 20 años (1). La incidencia de DM1 en Colombia en niños menores de 15 años es relativamente baja (de 3-4 por cada 100000 ) y la prevalencia se estima en un $0,07 \%(2,3)$.

La prueba de glucosa capilar es el método recomendado para el autocontrol de glucosa. Sin embargo, el dolor, la frecuencia y el miedo a la estigmatización son barreras comunes para la adherencia al seguimiento entre los adolescentes $(4,5)$. Se ha creado una nueva tecnología conocida como monitor flash de glucosa (MFG), lanzado al mercado en 2014. Su función principal es la medición continua de la concentración de glucosa en el líquido intersticial y la producción de un perfil de glucosa ambulatoria, que descarga mediante escaneo por Bluetooth $^{\circledR}$ las mediciones de glucosa del sensor al lector o a un dispositivo móvil $(5,6)$.

El objetivo de este documento es presentar una posición conjunta adoptada por un grupo de expertos especialistas en endocrinología pediátrica, quienes emitieron opiniones a partir de la experiencia y la evidencia revisada acerca del uso del MFG en pacientes menores de 18 años con DM1, con el fin de brindar recomendaciones en cuanto a las indicaciones, la frecuencia y el modo de uso del MFG, así como su perfil de seguridad y su impacto en la calidad de vida de los pacientes y cuidadores.

\section{Metodología}

La Asociación Colombiana de Endocrinología, Metabolismo y Diabetes (ACE) desarrolló un consenso con método Delphi modificado (7), definiendo como consenso positivo promedio $\geq 70 \%$, consenso negativo promedio $\leq 30 \%$ y disenso entre $31 \%$ y 69 \%. Para esto se estableció un grupo desarrollador y un panel de 8 expertos en el área de endocrinología pediátrica elegidos por un muestreo no probabilístico a conveniencia.

Se realizó una búsqueda sistemática en 3 bases de datos especializadas: Pubmed, ScienceDirect, Epistemonikos (Tabla 1) y se recibieron artículos aportados por el panel de expertos participantes del consenso. No hubo restricciones de idioma o de año de publicación. Posteriormente, se llevó a cabo el análisis de la evidencia científica mediante la herramienta del
Oxford Center for Evidence-Based Medicine: Levels of Evidence (March, 2009) (8).

Se formularon las preguntas a responder por el grupo de expertos, de forma anónima y en una plataforma online, mediante la escala de Likert de 1 a 9 (9). Se realizaron 3 sesiones de preguntas en las que los expertos expresaron comentarios acerca de sus experiencias y opiniones; posteriormente, se llevaron a cabo sesiones virtuales en las que, de forma conjunta y de acuerdo con los resultados de la mediana, se planteó la fuerza de recomendación y se definieron las recomendaciones.

\section{Resultados}

Los 8 participantes del consenso completaron la totalidad de preguntas realizadas en 3 rondas de encuestas. Después de cada ronda, tuvieron una retroalimentación controlada de los resultados, manteniendo el anonimato de las respuestas; en la primera ronda se presentaron 17 afirmaciones a analizar y se obtuvo consenso en 14/17. Se informaron los resultados a los participantes y se requirió una segunda ronda de iteración para profundizar en los temas en que se obtuvo disenso; se incluyeron 15 afirmaciones y se obtuvo consenso en 10/15 de estos planteamientos. Se realizó una tercera ronda de iteración, que incluyó un total de 8 afirmaciones, en la que se obtuvo consenso en 7/8; se plantearon las recomendaciones de acuerdo con las respuestas obtenidas y se presentaron en una reunión grupal final (Tablas 2, 3 y 4).

\section{Recomendaciones}

- Uso del MFG en pacientes menores de 18 años con diagnóstico de DM1:

1. Tiene una precisión comparable con la glucometría capilar y puede ser utilizado para el seguimiento clínico y la toma de decisiones. Existen circunstancias en las que la lectura debe verificarse con glucometría capilar, como en valores extremos de glucosa o en la lectura realizada en actividades asociadas con cambios rápidos de glucosa y en las que se deban tomar conductas; adicionalmente, ante la presencia de síntomas que no concuerden con la lectura obtenida con el monitor flash (fuerza de recomendación: fuerte).

2. Es una opción eficaz para controlar y generar una reducción significativa de la hemoglobina glucosilada $\left(\mathrm{HbA}_{1 \mathrm{c}} ; \geq 0,5 \%\right)$, incluyendo a los pacientes con control glucémico subóptimo $\left(\mathrm{HbA}_{1 \mathrm{c}}>7 \%\right)$; precisan el uso constante del dispositivo, con escaneos frecuentes y una adecuada adherencia a dieta y ejercicio (fuerza de recomendación: fuerte).

3. Mejora el tiempo en el rango en pacientes ambulatorios. Se recomienda su uso bajo vigilancia del médico especialista tratante (fuerza de recomendación: fuerte). 

Mellitus, Ketosis-Prone)) OR (Diabetes Mellitus, Sudden Onset)) OR (Diabetes, Autoimmune)) OR (Diabetes, Juvenile-Onset)) OR (Insulin Dependent Diabetes Mellitus 1)) AND (("child"[MeSH Terms] OR "adolescent"[MeSH Terms] OR ("child"[MeSH Terms] OR "child"[All Fields] OR "children"[All Fields] OR "child s"[All Fields] OR "children s"[All Fields] OR "children"[All Fields] OR"child"[All Fields]) OR ("adolescences"[All Fields] OR"adolescence"[All Fields] OR "adolescent"[MeSH Terms] OR "adolescent"[All Fields] OR "adolescence"[All Fields] OR "adolescents"[All Fields] OR "adolescent s"[All Fields]) OR ("adolescent"[MeSH Terms] OR "adolescent"[All Fields] OR "teen"[All Fields])) NOT "adult"[MeSH Terms])) AND ((((Blood Glucose Self-Monitoring[MeSH Terms]) OR (Free Style)) OR (Flash glucose monitoring system)) OR (Pro flash glucose monitoring system))) AND ((randomized controlled trial[pt] OR controlled clinical trial[pt] OR randomized[tiab] OR placebo[tiab] OR drug therapy[sh] OR randomly[tiab] OR trial[tiab] OR groups[tiab] NOT (animals [mh] NOT humans [mh]))) Filters: in the last 5 years

ScienceDirect $\quad$ Diabetes mellitus type 1 AND children AND adolescent AND Blood Glucose Self-Monitoring AND Flash glucose monitoring system - filter Research articles

Epistemonikos $\quad$ (title:((title:((title:((title:(Diabetes mellitus type 1) OR abstract:(Diabetes mellitus type 1)) OR (title:(Diabetes Mellitus, Insulin-Dependent) OR abstract:(Diabetes Mellitus, Insulin-Dependent)) OR (title:(Diabetes Mellitus, Juvenile Onset) OR abstract:(Diabetes Mellitus, Juvenile Onset)) OR (title:(Diabetes, Autoimmune) OR abstract:(Diabetes, Autoimmune))) OR abstract:((title:(Diabetes mellitus type 1) OR abstract:(Diabetes mellitus type 1)) OR (title:(Diabetes Mellitus, Insulin-Dependent) OR abstract:(Diabetes Mellitus, Insulin-Dependent)) OR (title:(Diabetes Mellitus, Juvenile Onset) OR abstract:(Diabetes Mellitus, Juvenile Onset)) OR (title:(Diabetes, Autoimmune) OR abstract:(Diabetes, Autoimmune)))) AND (title:((title:(children) OR abstract:(children)) OR (title:(adolescent) OR abstract:(adolescent))) OR abstract:((title:(children) OR abstract:(children)) OR (title:(adolescent) OR abstract:(adolescent))))) OR abstract:((title:((title:(Diabetes mellitus type 1) OR abstract:(Diabetes mellitus type 1)) OR (title:(Diabetes Mellitus, Insulin-Dependent) OR abstract:(Diabetes Mellitus, Insulin-Dependent)) OR (title:(Diabetes Mellitus, Juvenile Onset) OR abstract:(Diabetes Mellitus, Juvenile Onset)) OR (title:(Diabetes, Autoimmune) OR abstract:(Diabetes, Autoimmune))) OR abstract:((title:(Diabetes mellitus type 1) OR abstract:(Diabetes mellitus type 1)) OR (title:(Diabetes Mellitus, Insulin-Dependent) OR abstract:(Diabetes Mellitus, Insulin-Dependent)) OR (title:(Diabetes Mellitus, Juvenile Onset) OR abstract:(Diabetes Mellitus, Juvenile Onset)) OR (title:(Diabetes, Autoimmune) OR abstract:(Diabetes, Autoimmune)))) AND (title:((title:(children) OR abstract:(children)) OR (title:(adolescent) OR abstract:(adolescent))) OR abstract:((title:(children) OR abstract:(children)) OR (title:(adolescent) OR abstract:(adolescent)))))) AND (title:((title:(Blood Glucose Self-Monitoring) OR abstract:(Blood Glucose SelfMonitoring)) OR (title:(Free Style) OR abstract:(Free Style)) OR (title:(Flash glucose monitoring system) OR abstract:(Flash glucose monitoring system)) OR (title:(Pro flash glucose monitoring system) OR abstract:(Pro flash glucose monitoring system))) OR abstract:((title:(Blood Glucose Self-Monitoring) OR abstract:(Blood Glucose Self-Monitoring)) OR (title:(Free Style) OR abstract:(Free Style)) OR (title:(Flash glucose monitoring system) OR abstract:(Flash glucose monitoring system)) OR (title:(Pro flash glucose monitoring system) OR abstract:(Pro flash glucose monitoring system))))) OR abstract:((title:((title:((title:(Diabetes mellitus type 1) OR abstract:(Diabetes mellitus type 1)) OR (title:(Diabetes Mellitus, Insulin-Dependent) OR abstract:(Diabetes Mellitus, InsulinDependent)) OR (title:(Diabetes Mellitus, Juvenile Onset) OR abstract:(Diabetes Mellitus, Juvenile Onset)) OR (title:(Diabetes, Autoimmune) OR abstract:(Diabetes, Autoimmune))) OR abstract:((title:(Diabetes mellitus type 1) OR abstract:(Diabetes mellitus type 1)) OR (title:(Diabetes Mellitus, Insulin-Dependent) OR abstract:(Diabetes Mellitus, Insulin-Dependent)) OR (title:(Diabetes Mellitus, Juvenile Onset) OR abstract:(Diabetes Mellitus, Juvenile Onset)) OR (title:(Diabetes, Autoimmune) OR abstract:(Diabetes, Autoimmune)))) AND (title:((title:(children) OR abstract:(children)) OR (title:(adolescent) OR abstract:(adolescent))) OR abstract:((title:(children) OR abstract:(children)) OR (title:(adolescent) OR abstract:(adolescent))))) OR abstract:((title:((title:(Diabetes mellitus type 1) OR abstract:(Diabetes mellitus type 1)) OR (title:(Diabetes Mellitus, Insulin-Dependent) OR abstract:(Diabetes Mellitus, Insulin-Dependent)) OR (title:(Diabetes Mellitus, Juvenile Onset) OR abstract:(Diabetes Mellitus, Juvenile Onset)) OR (title:(Diabetes, Autoimmune) OR abstract:(Diabetes, Autoimmune))) OR abstract:((title:(Diabetes mellitus type 1) OR abstract:(Diabetes mellitus type 1)) OR (title:(Diabetes Mellitus, Insulin-Dependent) OR abstract:(Diabetes Mellitus, Insulin-Dependent)) OR (title:(Diabetes Mellitus, Juvenile Onset) OR abstract:(Diabetes Mellitus, Juvenile Onset)) OR (title:(Diabetes, Autoimmune) OR abstract:(Diabetes, Autoimmune)))) AND (title:((title:(children) OR abstract:(children)) OR (title:(adolescent) OR abstract:(adolescent))) OR abstract:((title:(children) OR abstract:(children)) OR (title:(adolescent) OR abstract:(adolescent)))))) AND (title:((title:(Blood Glucose Self-Monitoring) OR abstract:(Blood Glucose Self-Monitoring)) OR (title:(Free Style) OR abstract:(Free Style)) OR (title:(Flash glucose monitoring system) OR abstract:(Flash glucose monitoring system)) OR (title:(Pro flash glucose monitoring system) OR abstract:(Pro flash glucose monitoring system))) OR abstract:((title:(Blood Glucose Self-Monitoring) OR abstract:(Blood Glucose Self-Monitoring)) OR (title:(Free Style) OR abstract:(Free Style)) OR (title:(Flash glucose monitoring system) OR abstract:(Flash glucose monitoring system)) OR (title:(Pro flash glucose monitoring system) OR abstract:(Pro flash glucose monitoring system)))))) 
Tabla 2. Preguntas y resultados primera ronda

\section{Uso de MFG}

1. En cuanto a la precisión, la medición flash de glucosa es comparable con la medición de glucosa en sangre capilar en niños y adolescentes.

2. En cuanto a eficacia, el uso del MFG genera una reducción significativa de la $\mathrm{HbA}_{1 c}(\geq 0,5 \%)$.

3. En cuanto a eficacia, el uso del MFG mejora el tiempo en rango, en la población pediátrica ambulatoria.

4. El MFG es una opción eficaz para la monitorización de glucemia, en pacientes con control glucémico subóptimo $\left.\mathrm{HbA}_{1 c}>7 \%\right)$.

5. El MFG es eficaz para el control glucémico de pacientes pediátricos usuarios de bomba de insulina con sensor.

6. El MFG es eficaz para el control glucémico en pacientes pediátricos bajo vigilancia en la unidad de cuidado intensivo.

7. El uso de MFG para el seguimiento ambulatorio mejora el cumplimiento de la monitorización de glucosa por parte de los pacientes pediátricos.

8. El uso de MFG reduce el riesgo de episodios graves de hipoglucemia en la población pediátrica.

9. El MFG es efectivo para la detección de hipoglucemias no sintomáticas.

10. La precisión de la medición del dispositivo flash de glucosa permanece estable durante la actividad física, independientemente del nivel de intensidad.

\begin{tabular}{|l|l|l}
\hline 8,2 & 0,89 & 9,0 \\
\hline 7,9 & 1,36 & 8,0 \\
\hline 7,7 & 1,28 & 8,0 \\
\hline 8,2 & 0,71 & 8,0 \\
\hline 5,5 & 2,45 & 5,0 \\
\hline 8,4 & 2,0 & 5,0 \\
\hline 7,2 & 2,38 & 8,0 \\
\hline 7,4 & 2,33 & 8,0 \\
\hline 5,5 & 2,33 & 6,0 \\
\hline & & 9,0 \\
\hline
\end{tabular}

\section{Seguridad}

11. El uso del MFG se asocia con reacciones adversas leves (reacciones de hipersensibilidad local, dolor leve).

12. El uso del MFG no presenta reacciones adversas graves.

13. El manejo de las reacciones adversas frecuentes (reacciones de hipersensibilidad local, dolor leve) asociadas con el uso del dispositivo no requieren la suspensión del mismo.

\begin{tabular}{|c|c|c}
\hline 7,7 & 1,28 & 8,0 \\
\hline 9,0 & 0 & 9,0 \\
\hline 7,1 & 2,17 & 8,0 \\
\hline
\end{tabular}

\section{Calidad de vida}

14. Los usuarios del MFG experimentan satisfacción general con el funcionamiento del dispositivo.

15. El uso del MFG mejora la percepción de la calidad de vida de los pacientes.

16. El uso del MFG mejora la percepción de la calidad de vida de los padres de pacientes con diabetes tipo 1.

17. El uso del MFG reduce las descompensaciones de glucemia en pacientes menores de 18 años, lo que reduce el riesgo de hospitalización.

\begin{tabular}{|c|c|c}
\hline 8,6 & 0,74 & 9,0 \\
\hline 8,6 & 0,74 & 9,0 \\
\hline 8,7 & 0,46 & 9,0 \\
\hline 7,4 & 1,19 & 8,0 \\
\hline
\end{tabular}

4. Es eficaz para la detección de hipoglucemias no sintomáticas y puede reducir el riesgo de episodios de hipoglucemia grave en esta población (fuerza de recomendación: fuerte).
5. Reduce la presencia de hipoglucemias e hiperglucemias en pacientes menores de 18 años, lo cual podría reducir el riesgo de hospitalizaciones (fuerza de recomendación: fuerte). 
Tabla 3. Preguntas y resultados segunda ronda

Uso de MFG

1. El MFG es eficaz para el control glucémico de pacientes pediátricos usuarios de bomba de insulina sin sensor.

2. Con respecto a los pacientes pediátricos usuarios de bomba de insulina con sensor, no se requiere el uso del MFG.

3. Con respecto a los pacientes pediátricos usuarios de bomba de insulina con sensor, no está indicado el uso del MFG para la calibración del sensor.

4. El uso del MFG disminuye el número de punciones capilares generados por alarma del sensor, en pacientes pediátricos usuarios de bomba de insulina con sensor.

5. Los pacientes pediátricos usuarios de bomba de insulina con sensor deben calibrar el sensor de la bomba con punción capilar.

6. Con respecto al uso del MFG en pacientes pediátricos bajo vigilancia en la unidad de cuidado intensivo pediátrico no neonatal, se requieren estudios en población con diagnóstico de diabetes tipo 1 para establecer su eficacia.

7. Con respecto a la precisión de la medición del dispositivo flash de glucosa, permanece estable, independientemente de la temperatura y la humedad de la piel.

8. Con respecto a la precisión de la medición del dispositivo flash de glucosa, permanece estable durante la actividad física moderada (3-6 MET).

9. Con respecto a la precisión de la medición del dispositivo flash de glucosa, permanece estable durante la actividad física vigorosa (> $6 \mathrm{MET}$ ).

10. En cuanto a precisión, el MFG altera su medición cuando se presentan cambios rápidos en la glucemia del paciente.

\begin{tabular}{|c|c|c}
\hline 8,0 & 1,1 & 8,5 \\
\hline 8,1 & 1,3 & 8,5 \\
\hline 7,2 & 2,0 & 8,0 \\
\hline 5,6 & 2,4 & 6,0 \\
\hline 6,5 & 2,9 & 8,0 \\
\hline 7,1 & 1,9 & 8,0 \\
\hline 6,9 & 1,8 & 7,5 \\
\hline 7,1 & 2,4 & 8,0 \\
\hline 5,6 & 2,3 & 7,0 \\
\hline & 0,8 & 8,0 \\
\hline & & \\
\hline
\end{tabular}

\section{Frecuencia y modo de uso}

11. En los pacientes < 18 años con diabetes mellitus tipo 1, se recomienda el uso del MFG de forma permanente.

12. En los pacientes < 18 años con diabetes mellitus tipo 1, se recomienda el uso del MFG de forma intermitente, cada tres meses, durante 14 días.

13. Para obtener una visión global de la glucemia con el MFG, debe realizarse un escaneo al menos una vez cada 8 horas.

14. Con respecto al uso del MFG, se obtiene un mayor beneficio en cuanto a la disminución y prevención de hipoglucemia, cuando este es usado durante mínimo el 70 \% del día.

15. Para el éxito de la adherencia al uso del MFG es indispensable la educación al paciente y a sus padres, en cuanto a los beneficios, las reacciones adversas frecuentes y su abordaje.

\begin{tabular}{|c|c|c}
\hline 8,2 & 0,7 & 8,0 \\
\hline 3,4 & 1,7 & 4,0 \\
\hline 7,7 & 1,9 & 8,5 \\
\hline 8,6 & 0,7 & 9,0 \\
\hline 8,7 & 0,7 & 9,0 \\
\hline
\end{tabular}

6. Está indicado para el seguimiento ambulatorio, lo que puede mejorar el cumplimiento de la monitorización de la glucosa y la adherencia (fuerza de recomendación: fuerte).

7. Para establecer recomendaciones acerca del uso del MFG en pacientes menores de 18 años con diagnóstico de DM1 y bajo vigilancia en la unidad de cuidado intensivo pediátrico (UCIP) no neonatal se requiere el desarrollo de estudios clínicos (fuerza de recomendación: fuerte).

- MFG en usuarios de bomba de insulina sin sensor:

8. Puede ser eficaz para el control glucémico de pacientes usuarios de bomba de insulina no acoplada a sensor. Existen circunstancias, explicadas previamente, en las 
Tabla 4. Preguntas y resultados segunda ronda

\begin{tabular}{|c|c|c|c|}
\hline Afirmación & Media & DE & Mediana \\
\hline \multicolumn{4}{|l|}{ Indicaciones de uso en usuarios de bomba de insulina } \\
\hline $\begin{array}{l}\text { 1. En pacientes pediátricos usuarios de bomba de insulina con sensor que requiera calibración, } \\
\text { es útil el uso del MFG para determinar la glucometría preprandial. }\end{array}$ & 3,0 & 2,6 & 2,0 \\
\hline $\begin{array}{l}\text { 2. En pacientes pediátricos usuarios de bomba de insulina con sensor que requiera calibración, } \\
\text { es útil el uso de glucometría capilar para determinar la glucometría preprandial. }\end{array}$ & 8,9 & 0,3 & 9,0 \\
\hline $\begin{array}{l}\text { 3. En pacientes pediátricos usuarios de bomba de insulina con sensor que requiera calibración, } \\
\text { es útil el uso de glucometría capilar para la calibración del sensor. }\end{array}$ & 8,9 & 0,3 & 9,0 \\
\hline $\begin{array}{l}\text { 4. En pacientes pediátricos usuarios de bomba de insulina con sensor que requiera calibración, } \\
\text { es útil el uso del MFG para la calibración del sensor. }\end{array}$ & 2,2 & 1,9 & 1,5 \\
\hline $\begin{array}{l}\text { 5. En pacientes pediátricos usuarios de bomba de insulina con sensor que requiera calibración, } \\
\text { es útil el uso de glucometría capilar para confirmar la alarma del sensor. }\end{array}$ & 8,7 & 0,4 & 9,0 \\
\hline $\begin{array}{l}\text { 6. En pacientes pediátricos usuarios de bomba de insulina acoplada con sensor que requiera } \\
\text { calibración, es útil el uso del MFG para confirmar la alarma del sensor. }\end{array}$ & 2,0 & 1,6 & 1,5 \\
\hline $\begin{array}{l}\text { 7. En pacientes pediátricos con diagnóstico de diabetes tipo 1, durante eventos que produzcan } \\
\text { cambios rápidos de glucosa, es confiable el uso del MFG. }\end{array}$ & 5,0 & 2,6 & 7,0 \\
\hline $\begin{array}{l}\text { 8. En pacientes pediátricos usuarios de bomba de insulina acoplada con sensor que requiera } \\
\text { calibración, se requieren más estudios para establecer las ventajas y desventajas del uso del } \\
\text { MFG. }\end{array}$ & 7,2 & 2,8 & 9,0 \\
\hline
\end{tabular}

que la lectura debe verificarse con glucometría capilar (fuerza de recomendación: fuerte).

- MFG en usuarios de insulina con sensor que requiera calibración:

9. La determinación de la glucosa preprandial debe realizarse con glucometría capilar. No se recomienda el uso del MFG para esta medición (fuerza de recomendación: fuerte).

10. La calibración del sensor de la bomba se realiza con glucometría capilar. No se recomienda el uso del MFG (fuerza de recomendación: fuerte).

11. Para la confirmación de las alarmas emitidas por el sensor se recomienda el uso de glucometría capilar. No se recomienda el uso del MFG con este fin (de acuerdo con el criterio del médico especialista tratante).

12. En usuarios de bomba de insulina acoplada con sensor que requiera calibración se requieren más estudios para establecer las ventajas y desventajas del uso del MFG (fuerza de recomendación: fuerte).

- Uso del MFG y la realización de actividad física:

13. La precisión del MFG permanece estable durante la realización de actividad física moderada (3-6 MET) (fuerza de recomendación: fuerte).
14. En relación con la actividad vigorosa, se encontró disenso. No se deben tomar acciones basadas en cifras de glucosa sin confirmar con glucometría capilar (fuerza de recomendación: fuerte).

15. Si durante la realización de actividad física se presenta sintomatología sugestiva de hipoglucemia o hiperglucemia, o valores extremos de glucosa, se debe confirmar con glucometría capilar (fuerza de recomendación: fuerte).

- Frecuencia y modo de uso del MFG:

16. Se debe hacer uso continuo del MFG y la realización de escaneo al menos 1 vez cada 8 horas para obtener una visión global de la glucemia del paciente (fuerza de recomendación: fuerte).

17. Hay mayor beneficio en cuanto a la disminución y prevención de la hipoglucemia cuando el MFG se usa durante mínimo el 70 \% del tiempo (fuerza de recomendación: fuerte, por lo cual no recomiendan el uso del MFG de forma intermitente [interrupción cada 14 días, 3 meses, etc.], debido a que disminuye el beneficio en la reducción de HbA1c y de episodios de hipoglucemia e hiperglucemia. De acuerdo con el criterio del médico especialista tratante). 
- En cuanto a la calidad de vida y el uso del MFG, los expertos consideran que:

18. Los usuarios del MFG y sus padres experimentan satisfacción general con el funcionamiento del dispositivo y la mejoría de la percepción en cuanto a calidad de vida (fuerza de recomendación: fuerte).

- Seguridad:

19. Es seguro y no se asocia con el desarrollo de reacciones adversas graves (fuerza de recomendación: fuerte).

20. Se asocia con reacciones adversas locales leves (hipersensibilidad local, dolor leve), que pueden ser manejadas con medidas locales y aislamiento del adhesivo (fuerza de recomendación: fuerte).

21. Para el éxito de la adherencia al uso del MFG es indispensable la educación al paciente y a sus padres en cuanto a los beneficios, las reacciones adversas y su abordaje (fuerza de recomendación: fuerte).

\section{Discusión}

La aprobación del MFG para su utilización en niños mayores de 4 años y su comercialización en Colombia ha generado grandes expectativas. En cuanto a la precisión clínica del MFG en comparación con la glucometría capilar, Edge y colaboradores describieron la exactitud al comparar los valores del MFG con las glucemias capilares. Se obtuvo un $83,8 \%$ de resultados en zona $\mathrm{A}$ y un $99,4 \%$ en zonas A y B, lo que demuestra una buena correlación entre el sensor y los niveles de glucemia capilar, con un coeficiente de correlación de 0,95 (6). En 2018 Hansen y colaboradores informaron que el MFG tiene una precisión clínica satisfactoria, en comparación con la glucometría capilar, con un 98,8 \% de los valores dentro de las zonas clínicamente aceptables (10). Así mismo, Pintus y colaboradores (11), y Landau y colaboradores (12) mostraron una mejoría significativa y sostenida de la $\mathrm{HbA}_{1 \mathrm{c}}$ en pacientes con el uso de este dispositivo. La utilidad del MFG en ejercicio está supeditada a los cambios fisiológicos que se generan durante la actividad física (13).

Se considera de gran utilidad el uso del MFG en pacientes con hipoglucemias frecuentes y asintomáticas. En concordancia con el estudio de Deeb y colaboradores, donde se comparó la glucometría capilar frente al MFG, se observó una diferencia significativa en la detección de hipoglucemia nocturna y diurna entre ambos, favoreciendo el MFG (14).

En los usuarios de bomba de insulina no acoplada a sensor, el MFG mostró con significancia estadística menos eventos de hipoglucemia y un menor temor a hipoglucemias, generando acciones preventivas para evitarlas (5). En pacientes usuarios de bomba con sensor se discutió ampliamente. Aunque son pocos los estudios que comparan el MFG con la bomba acoplada al sensor (BAS), Massa y colaboradores (2019) no encontraron diferencias en relación con la $\mathrm{HbA}_{1 c}$, pero sí en cuanto a la variabilidad glucémica y al tiempo en hipoglucemia a favor de la BAS, con el inconveniente de la calibración por punción capilar y las alarmas y su respectiva confirmación por punción capilar (15). La BAS es la opción más apropiada, aunque considerando las preferencias del paciente se puede tomar en cuenta la opción de MFG (16).

Se ha demostrado que, a mayor frecuencia de mediciones del MFG, mejor será el impacto glucémico (17). Al escanear con el sistema, este mostrará los datos tomados y almacenados por el sensor cada 15 minutos durante las últimas 8 horas (18). Se recomienda realizar una medición antes de cumplir este intervalo de tiempo.

El MFG es un dispositivo seguro y su uso está asociado con reacciones adversas leves, como hipersensibilidad local y dolor leve, que pueden ser manejadas con medidas locales y con aislamiento del adhesivo. El estudio IMPACT informó de 13 eventos adversos relacionados con el dispositivo que estaban relacionados con el uso del sensor y se clasificaron como leves (3), moderados (4) y graves (6). Se resolvieron mediante el uso de productos de barrera o terapia con medicamentos tópicos (19). Con el fin de asegurar el éxito de la adherencia, juega un papel importante la educación al paciente y a sus padres en cuanto a los beneficios, las reacciones adversas y su abordaje.

Se evaluó el impacto en la calidad de vida, en la que hubo acuerdo entre los expertos acerca del potencial del sistema MFG en el mejoramiento de la calidad de vida de los pacientes y de sus cuidadores. Hayek y colaboradores (2018) publicaron un estudio en el que se evaluó la calidad de vida y el miedo a la hipoglucemia. Se aplicó el cuestionario PedsQL 3.0 y HFS-C, antes del inicio del MFG y a 3 meses de su uso. Los resultados evidenciaron que todos los indicadores, tanto de la escala de calidad de vida como del miedo a la hipoglucemia, mejoraron de forma estadísticamente significativa luego de 3 meses de uso del MFG (5). Vergier y colaboradores (2019) publicaron un estudio multicéntrico cuyo objetivo fue establecer las opiniones de pacientes pediátricos con diagnóstico de DM1 sobre el uso diario del MFG. El 89,5 \% informó cambios en sus hábitos, el 70,6 \% tomó más mediciones glucométricas, el 37,2 \% corrigió su hiperglucemia rápidamente y el 37,5 \% utilizó las tendencias para ajustar sus dosis de insulina (20).

\section{Conclusiones}

El MFG de primera generación es una tecnología innovadora para el control glucémico de pacientes menores de 18 años con diagnóstico de DM1. Tiene una precisión comparable con la glucometría capilar y se considera un dispositivo seguro con la capacidad de impactar positivamente en la calidad de vida de los pacientes y sus cuidadores. La confirmación del valor con glucometría capilar está indicada en pacientes con glucometrías extremas, en momentos de cambios rápidos de glucosa en los que se deban tomar conductas y, adicionalmente, cuando los síntomas no concuerden con la lectura obtenida. El 
MFG es un dispositivo seguro, no asociado con reacciones adversas graves; ocasionalmente, se ha asociado con reacciones adversas leves que responden adecuadamente a un manejo tópico o de medidas locales.

\section{Fuentes de financiación}

Este consenso fue financiado por la Asociación Colombiana de Endocrinología, Diabetes y Metabolismo (ACE), que no participó en el diseño del estudio, en la recopilación o el análisis de los datos, ni en la realización del manuscrito. Esta cooperación fue recibida por el grupo desarrollador Neurobusiness $^{\circledR}$, por parte de la Dra. María Angélica García Sánchez, médica epidemióloga, y la Dra. Carolina Munar Fonseca, médica epidemióloga.

\section{Conflictos de interés}

Los participantes de este consenso recibieron honorarios de la ACE.

\section{Referencias}

1. Patterson CC, Karuranga S, Salpea P, Saeedi P, Dahlquist G, Soltesz G, et al. Worldwide estimates of incidence, prevalence and mortality of type 1 diabetes in children and adolescents: Results from the International 12. Diabetes Federation Diabetes Atlas, 9. ${ }^{a}$ edición. Diabetes Res Clin Pract. 2019;157:107842.

2. Atlas de la Diabetes de la FID. 9 a edición. Federación Internacional de Diabetes; 2019.

3. Aschner P. Epidemiología de la diabetes en Colombia. Av Diabetol. 2010;26(2):95-100.

4. Boucher SE, Gray AR, de Bock M, Wiltshire EJ, Galland BC, Tomlinson PA, et 14 al. Effect of 6 months' flash glucose monitoring in adolescents and young adults with type 1 diabetes and suboptimal glycaemic control: managing diabetes in a 'flash' randomised controlled trial protocol. BMC Endocr Disord. 2019;19(1):50.

5. Al Hayek AA, Robert AA, Al Dawish MA. Evaluation of FreeStyle Libre Flash Glucose Monitoring System on Glycemic Control, Health-Related Quality of Life, and Fear of Hypoglycemia in Patients with Type 1 Diabetes. Clin Med Insights Endocrinol Diabetes. 2017;10:1179551417746957.

6. Edge J, Acerini C, Campbell F, Hamilton-Shield J, Moudiotis C, Rahman S, et al. An alternative sensor-based method for glucose monitoring in children and young people with diabetes. Arch Dis Child. 2017;102(6):543-549.

7. Boulkedid R, Abdoul H, Loustau M, Sibony O, Alberti C. Using and reporting 17. the Delphi method for selecting healthcare quality indicators: a systematic review. PLoS One. 2011;6(6):e20476.

8. Manterola DC, Zavando MD. Cómo interpretar los "Niveles de Evidencia" en los diferentes escenarios clínicos. Rev Chil Cir. 2009;61(6):582-95.

9. Matas A, Matas A. Diseño del formato de escalas tipo Likert: un estado de la cuestión. Rev Electrónica Investig Educ. 2018;20(1):38-47.

10. Hansen EA, Klee P, Dirlewanger M, Bouthors T, Elowe-Gruau E, Stoppa- 19. Vaucher S, et al. Accuracy, satisfaction, and usability of a flash glucose monitoring system among children and adolescents with type 1 diabetes 20 . attending a summer camp. Pediatr Diabetes. 2018;19(7):1276-84.

11. Pintus D, Ng SM. Freestyle libre flash glucose monitoring improves patient quality of life measures in children with Type 1 diabetes mellitus (T1DM) with appropriate provision of education and support by healthcare professionals. Diabetes Metab Syndr. 2019;13(5):2923-6.

Landau Z, Abiri S, Gruber N, Levy-Shraga Y, Brener A, Lebenthal Y, et al. Use of flash glucose-sensing technology (FreeStyle Libre) in youth with type 1 diabetes: AWeSoMe study group real-life observational experience. Acta Diabetol. 2018;55(12):1303-10.

3. Moser O, Yardley JE, Bracken RM. Interstitial Glucose and Physical Exercise in Type 1 Diabetes: Integrative Physiology, Technology, and the Gap InBetween. Nutrients. 2018;10(1):93.

Deeb A, Yousef H, Al Qahtani N, Artan I, Suliman S, Tomy M, et al. Nove ambulatory glucose-sensing technology improves hypoglycemia detection and patient monitoring adherence in children and adolescents with type 1 diabetes. J Diabetes Metab Disord. 2019;18(1):1-6.

15. Massa GG, Gys I, Bevilacqua E, Wijnands A, Zeevaert R. Comparison of flash glucose monitoring with real time continuous glucose monitoring in children and adolescents with type 1 diabetes treated with continuous subcutaneous insulin infusion. Diabetes Res Clin Pract. 2019;152:111-8.

16. Campbell FM, Murphy NP, Stewart C, Biester T, Kordonouri O. Outcomes of using flash glucose monitoring technology by children and young people with type 1 diabetes in a single arm study. Pediatr Diabetes. 2018;19(7):1294-301.

Dunn TC, Xu Y, Hayter G, Ajjan RA. Real-world flash glucose monitoring patterns and associations between self-monitoring frequency and glycaemic measures: A European analysis of over 60 million glucose tests. Diabetes Res Clin Pract. 2018;137:37-46.

18. Pleus S, Kamecke U, Link M, Haug C, Freckmann G. Flash Glucose Monitoring: Differences Between Intermittently Scanned and Continuously Stored Data. J Diabetes Sci Technol. 2018;12(2):397-400.

Leelarathna L, Wilmot EG. Flash forward: a review of flash glucose monitoring. Diabet Med J Br Diabet Assoc. 2018;35(4):472-82.

Vergier J, Samper M, Dalla-Vale F, Ventura V, Baucher F, Joubert F, et al. Evaluation of flash glucose monitoring after long-term use: A pediatric survey. Prim Care Diabetes. 2019;13(1):63-70. 


\section{SÍNDROME DE \\ QUILOMICRONEMIA FAMILIAR (FCS):}

Hereditario. Un cuadro clínico doloroso, recurrente y potencialmente fatal. A menudo subdiagnosticado.

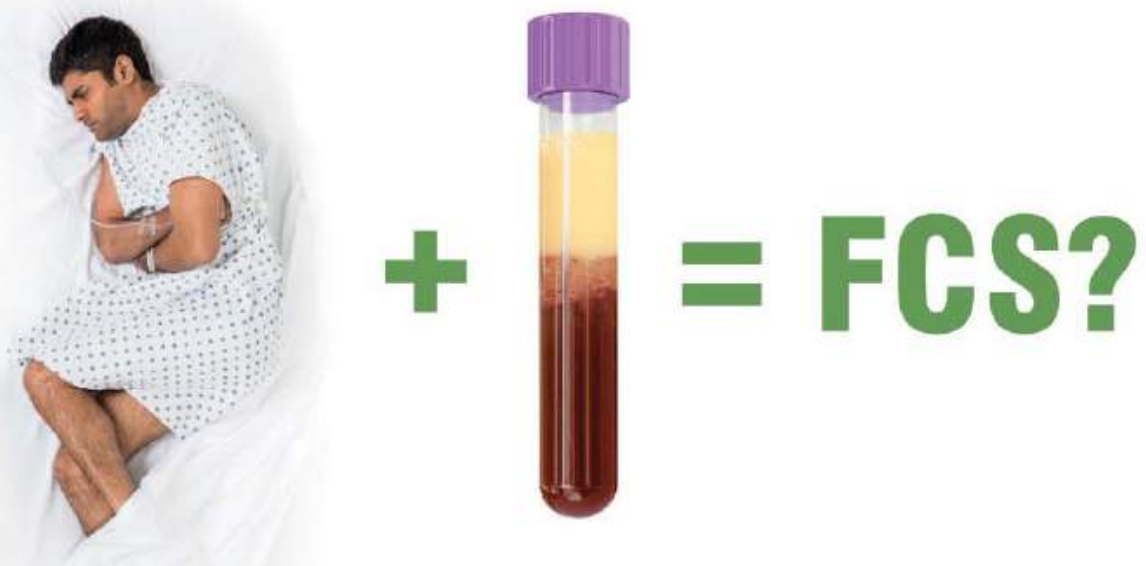

\section{Qué es FCS?}

- EI FCS es una enfermedad genética de disfunción enzimática que inhibe el metabolismo de los quilomicrones. ${ }^{2,4}$

- La acumulación consiguiente de quilomicrones conduce a hipertrigliceridemia severa y las complicaciones asociadas. ${ }^{5}$

- El FCS es una condición que expone a los pacientes al riesgo de pancreatitis aguda, cuadro potencialmente fatal, así como a múltiples morbilidades a largo plazo, tales como dolor abdominal crónico y diabetes pancreatogénica. 1,2,5,6

\section{EL FCS PUEDE SER DIAGNOSTICADO CLÍNICAMENTE. BUSCAR: ${ }^{\circ}$}

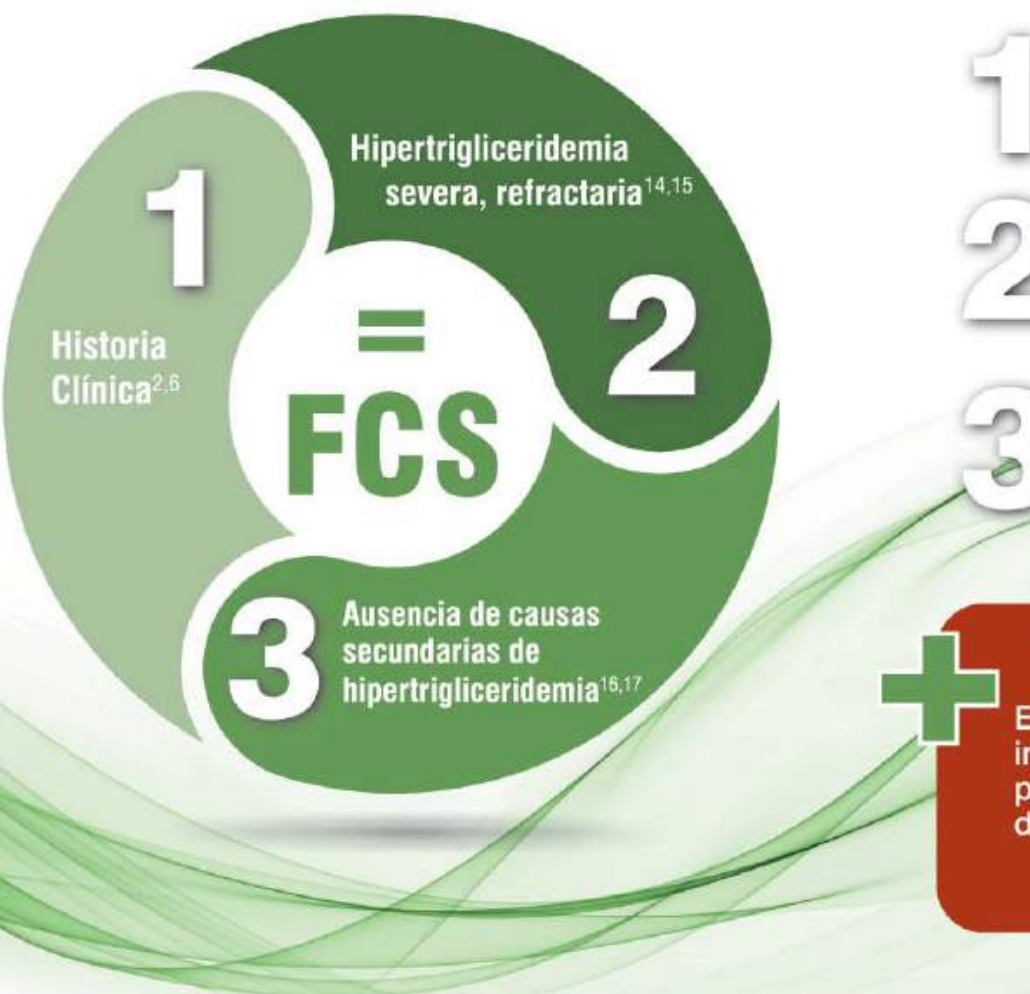

- Pancreatitis aguda y / o dolor abdominal (puede ser recurrente o crónico), sin otras causas explicables

- Triglicéridos en ayunas $>880 \mathrm{mg} / \mathrm{dL}$

- Sin respuesta, respuesta mínima a las terapias estándar.

- Exceso de consumo de alcohol diabetes no controlada, ingesta de ciertos medicamentos $u$ otras condiciones médicas.

\section{EL ESTUDIO GENÉTICO ES ESENCIAL}

En caso tenga una sospecha o requiera mayor información. La toma de muestra será programada por el laboratorio Gencell Pharma en los laboratorios de referencia de su ciudad:

pacientes@labgencell.com

línea nacional $3183314900 / 3166904882$

a: Otros nombres para el FCS incluyen deficiencia de lipoprotein lipasa, hiperlipoproteinemia tipo I e hiperlipidemia familliar. ${ }^{24,1213}$ b. Criterio diagnóstico propuesto

Relerencias:

1. Davidson et al. Excert hev Cardiovasc Ther. 2017:15(5):415-423. 2. Brahm et a. Nat Rev Endocrnol 2015:11:352-362. 3. Chat et al. Advances in Internal Medicine. 1992:249-273.

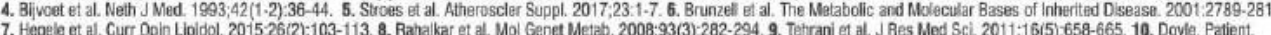

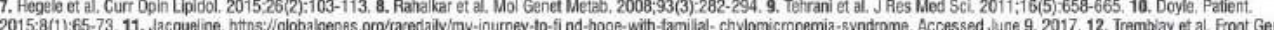

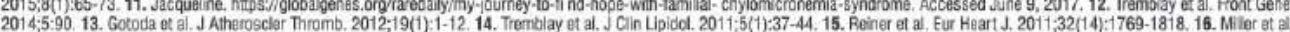
Circulation. 2011:123(20):2292-2333. 17. Berglund et al. J Clin Endocrinol Metab. 2012:97(9):2969-2989. 18. Nawaz et a. Am J Gastroenterol, 2015:110(10):1497-1503

Contenido desarrollado exciusivamente para profesionales de la salud

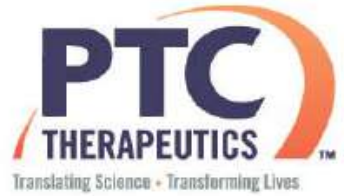

